\title{
ELEMENTARY OPERATORS ON PRIME C*-ALGEBRAS II $\dagger$
}

\author{
by MARTIN MATHIEU $\ddagger$
}

(Received 18 April, 1987)

1. Introduction. Compact elementary operators acting on the algebra $\mathscr{L}(H)$ of all bounded operators on some Hilbert space $H$ were characterised by Fong and Sourour in [9]. Akemann and Wright investigated compact and weakly compact derivations on $\mathrm{C}^{*}$-algebras [1], and also studied compactness properties of the sum and the product of the right and the left regular representation of an element in a $\mathrm{C}^{*}$-algebra [2]. They used the concept of a compact Banach algebra element due to Vala [17]: an element $a$ in a Banach algebra $A$ is called compact if the mapping $x \mapsto a x a$ is compact on $A$. This notion has been further investigated by Ylinen $[18, \mathbf{1 9}, \mathbf{2 0}]$, who showed in particular that $a$ is a compact element of the $\mathrm{C}^{*}$-algebra $A$ if $x \mapsto \operatorname{axa}$ is weakly compact on $A$ [19].

In Section 2 we will discuss some of the properties of the set $K(A)$ of compact elements in a $C^{*}$-algebra $A$ with emphasis on the case of a prime $\mathrm{C}^{*}$-algebra. This information will then be used in Section 3 to characterise compact and weakly compact elementary operators on prime $C^{*}$-algebras which extends the results in [2] and [9]. As a consequence we will confirm a conjecture by Fong and Sourour that on the Calkin algebra there do not exist non-zero compact elementary operators in a much stronger form.

An elementary operator on a $C^{*}$-algebra $A$ is a mapping of the form $S: x \mapsto \sum_{j=1}^{n} a_{j} x b_{j}$, where $a=\left(a_{1}, \ldots, a_{n}\right), b=\left(b_{1}, \ldots, b_{n}\right) \in M(A)^{n}$ and $M(A)$ denotes the multiplier algebra of $A$. It has emerged that the properties of $S$ can be described very neatly if the algebra $A$ is prime; i.e., if the product $I J$ of any two non-zero ideals $I$ and $J$ of $A$ is again non-zero. More generally, an ideal $I$ of $A$ is a prime ideal if the quotient $A / I$ is a prime algebra. For example the ideal $\mathscr{K}(H)$ of all compact operators on a Hilbert space $H$ is a prime ideal in $\mathscr{L}(H)$.

The main tool to obtain the above mentioned characterisations, Theorems 3.7 and 3.8 , will be the following result which was proved in the first part of the paper ([13, Theorem 4.1]).

THEOREM 1.1. Let $A$ be a prime $C^{*}$-algebra and for $a, b \in M(A)^{n}$ let $S=\sum_{j=1}^{n} M_{a_{j}, b_{j}}$ be the corresponding elementary operator. If the set $\left\{b_{1}, \ldots, b_{n}\right\}$ is linearly independent, then the following conditions are equivalent:
(a) $S=0$,
(b) $a_{j}=0$ for $1 \leqq j \leqq n$.

$\dagger$ The results in this paper are based on results in the author's doctoral thesis at the University of Tübingen, West Germany.

$\ddagger$ Research supported in part by DFG.

Glasgow Math. J. 30 (1988) 275-284. 
This result gives full information in which way the elements $a_{j}, b_{j} \in M(A)$ can be changed without changing the generated elementary operator. In order to make this more explicit, we call the elementary operator $x \mapsto a x b, \quad(a, b \in M(A))$, a (two-sided) multiplication on $A$ and denote it by $M_{a, b}$. The minimal length $l(S)$ of a non-zero elementary operator $S$ now is the minimum of the number of multiplications whose sum represents $S$. In the case $S=0$ we define $l(S)=0$. Each representation of $S$ by a sum of $l(S)$ multiplications is called a minimal representation of $S$. As an immediate consequence of Theorem 1.1, a representation $S=\sum_{j=1}^{n} M_{a_{j}, b_{j}}$ is minimal if and only if both $\left\{a_{1}, \ldots, a_{n}\right\}$ and $\left\{b_{1}, \ldots, b_{n}\right\}$ are linearly independent subsets of $M(A)$, and in any other minimal representation $S=\sum_{k=1}^{n} M_{c_{k}, d_{k}}$ the elements $c_{k}, d_{k}$ are linear combinations of $a_{j}, b_{j}$ respectively whenever $A$ is a prime $C^{*}$-algebra ([13, Proposition 4.6 and Corollary 4.7]).

2. The ideal of compact elements in a prime $\mathbf{C}^{*}$-algebra. Throughout this paper $A$ will denote a $C^{*}$-algebra with spectrum $\hat{A}$. The reduced atomic representation $\pi_{a}$ of $A$ is the direct sum $\pi_{a}=\bigoplus_{t \in \hat{A}} \pi_{t}$, where $\left(\pi_{t}, H_{t}\right)$ is some irreducible representation in $t \in \hat{A}$ (cf. $[15,4.3])$. If $\left\{A_{i} \mid i \in I\right\}$ is a family of $\mathrm{C}^{*}$-algebras, by $\sum_{i}^{\oplus} A_{i}$ we will denote their restricted direct sum, i.e.

$$
\sum_{i}^{\oplus} A_{i}=\left\{x=\left(x_{i}\right) \in \sum_{i}^{\oplus} A_{i} \mid \forall \varepsilon>0:\left\{i \in I \mid\left\|x_{i}\right\| \geqq \varepsilon\right\} \text { is finite }\right\} .
$$

$\mathrm{A} \mathrm{C}^{*}$-algebra is said to be of elementary type if it is isomorphic to $\mathscr{K}(H)$ for some Hilbert space $H$. The socle $\operatorname{soc}(A)$ of a $\mathrm{C}^{*}$-algebra $A$ is the sum of all minimal left ideals of $A$, unless $A$ does not contain any minimal left ideal in which case $\operatorname{soc}(A)=0$. The elements in $\operatorname{soc}(A)$ are called finite rank elements; it is easily seen that $a \in \operatorname{soc}(A)$ if and only if $M_{a, a}$ is a finite rank operator on $A\left(\left[5, C^{*} .1 .2\right]\right)$.

The following proposition, entailing in particular that $K(A)$ is a closed ideal, lists several known characterisations of the compact elements of a $\mathrm{C}^{*}$-algebra $A$. The equivalences (b) $\Leftrightarrow(c) \Leftrightarrow(e)$ are implicit in [2], and (b) $\Leftrightarrow(c)$ is [19, Theorem 3.1]. Here, they will follow from our results on elementary operators; thus the proof is postponed until Section 3.

Proposition 2.1. The following conditions on an element $a$ of a $\mathrm{C}^{*}$-algebra $A$ are equivalent:

(a) $a \in K(A)$;

(b) $M_{a, a}$ is compact;

(c) $M_{a, a}$ is weakly compact;

(d) $a \in \operatorname{soc}(A)$;

(e) $a \in \sum_{n}^{\oplus} I_{n}$, where $\left(I_{n}\right)_{n \in \mathbb{N}}$ is a sequence of orthogonal closed ideals of $A$ of elementary type;

(f) $\pi_{a}(a) \in \sum_{t}^{\oplus} \mathscr{K}\left(H_{t}\right)$ 
In the case where $A$ is equal to $\mathscr{L}(H)$, the ideal $K(A)$ coincides with $\mathscr{K}(H)$ ([16, Theorem 3]), and thus $K(A)$ is the unique minimal closed ideal of $A$. This remains true in prime $\mathrm{C}^{*}$-algebras.

Proposition 2.2. If $A$ is a prime $\mathrm{C}^{*}$-algebra then $\operatorname{soc}(A)$ is contained in every non-zero closed ideal of $A$. Thus, if $K(A) \neq 0$, it is the only minimal closed ideal of $A$.

Proof. Let $e$ be a minimal projection in $A$. Then $P_{e}=\{x \in A \mid x A e=0\}$ is the unique primitive ideal in $A$ which does not contain $e([5, \mathrm{BA} .3 .5])$. Since $A$ is prime, $P_{e}=0$. Indeed, $A x A A e A=0$ implies that $A x A=0$. Thus every non-zero primitive ideal $P$ of $A$ contains $e$, and since every minimal left ideal $L$ is of the form $L=A e$ for some minimal projection $e$, it follows that $\operatorname{soc}(A) \subseteq P$. Since each closed ideal of $A$ is the intersection of those primitive ideals which contain it $([15,3.13 .8]), \operatorname{soc}(A)$ is contained in every non-zero closed ideal. Since $K(A)=\overline{\operatorname{soc}(A)}$ by 2.1 , the second assertion follows.

We now investigate the structure of $K(A)$ for a prime $C^{*}$-algebra $A$. For the notions of liminal, antiliminal, and type $I \mathrm{C}^{*}$-algebras see [7, Section 4] and [15, Chapter 6]. We recall here that a positive element $a$ in a $C^{*}$-algebra $A$ is called abelian if the range of the multiplication $M_{a, a}$ is a commutative algebra. A $C^{*}$-algebra which is generated by its abelian elements is said to be of type $I_{0}$. Every $C^{*}$-algebra contains a largest ideal of type $\mathrm{I}_{0}$, namely the $\mathrm{C}^{*}$-subalgebra $I_{\text {abel }}$ generated by all abelian elements $([15,6.1 .7])$.

Proposition 2.3. If $A$ is a prime $\mathrm{C}^{*}$-algebra, then $K(A)=I_{\mathrm{abel}}$. In particular, a prime $C^{*}$-algebra $A$ is antiliminal if and only if $K(A)=0$.

Proof. If $A$ does not contain any non-zero abelian element, then there is no minimal projection in $A$ whence $K(A)=I_{\text {abel }}=0$. Thus we may assume that $A$ is not antiliminal. Then, by Proposition 2.2, $K(A) \subseteq I_{\text {abel }}$. To prove the reverse inclusion take a non-zero abelian element $a$ in $A$. Then

$$
\text { axaaya =ayaaxa, (for all } x, y \in A \text { ). }
$$

Thus $\left(a^{2} x a^{2}\right) y a^{2}-a^{2} y\left(a^{2} x a^{2}\right)=0$, for all $x, y \in A$. Since $A$ is prime, by Theorem 1.1, for each $x \in A$ there is $\lambda(x) \in \mathbb{C}$ such that $a^{2} x a^{2}=\lambda(x) a^{2}$. In particular, $M_{a^{2}, a^{2}} A \subseteq \mathbb{C} a^{2}$. Since $a^{6}=\lambda\left(a^{2}\right) a^{2}$ with $\lambda\left(a^{2}\right)>0$, the element $e:=\lambda\left(a^{2}\right)^{-1} a^{4}$ is a projection in $A$. Thus, $a=\lambda\left(a^{2}\right)^{1 / 4} e$ is a scalar multiple of a minimal projection for

$$
e A e=a^{2} A a^{2} \subseteq \mathbb{C} a^{2}=\mathbb{C} e .
$$

This proves $I_{\mathrm{abel}} \subseteq K(A)$.

Remarks. 1. Since a prime $\mathrm{C}^{*}$-algebra which contains minimal projections is primitive (see below), it follows from Proposition 2.3 that a prime $\mathrm{C}^{*}$-algebra which is not antiliminal is primitive. This, of course, is well known (cf. e.g. [4, Lemma 3.1]); however, our approach via elementary operators reveals that this is only a special case of Martindale's characterisation of prime rings satisfying a generalised polynomial identity ([12, Theorem 3]). 
2. The inclusion $K(A) \subseteq I_{\text {abcl }}$ remains true without primeness. In fact, for every $C^{*}$-algebra $A$ the ideal $K(A)$ is of type $\mathrm{I}_{0}([14,6.14 .3])$. Therefore, $K(A)$ is liminal and thus contained in the largest liminal ideal $I_{\text {lim }}$ of $A$ (cf. [18, p. 32]). A commutative $\mathrm{C}^{*}$-algebra without minimal projections provides an example of a liminal $\mathrm{C}^{*}$-algebra with $K(A)=0$. It is easy to see that, for a prime $\mathrm{C}^{*}$-algebra $A$, again $K(A)$ coincides with $I_{\text {lim }}$.

A W*-algebra is prime if and only if it is a factor. In addition, each closed ideal in a factor is a prime ideal. The following well-known result shows in particular that, in every factor $A$, the ideal $K(A)$ is also the largest ideal of type $\mathrm{I}$.

Proposition 2.4. For every Hilbert space $H$ the Calkin algebra $\mathscr{C}(H)=\mathscr{L}(H) / \mathscr{K}(H)$ is an antiliminal prime $\mathrm{C}^{*}$-algebra.

For an arbitrary prime $C^{*}$-algebra $A$ the situation may be different. Apart from trivial cases, e.g. if $A$ is liminal or antiliminal, the generalised Calkin algebra $A / K(A)$ need neither be antiliminal nor be prime. For an example let $A$ be the $C^{*}$-algebra generated by $\mathscr{K}(H)$ and two orthogonal infinite dimensional projections on $H$. Then $A / K(A) \cong \mathbb{C}^{2}$.

Nevertheless, primeness will play an important role in the next section. Before we turn our attention back to elementary operators, we recall that a prime $\mathrm{C}^{*}$-algebra $A$ with non-zero socle is already primitive. If $p$ is a minimal projection in $A$, we endow the minimal left ideal $A p$ with an inner product $(x \mid y)=y^{*} x$ and put $H_{p}=(A p,(\cdot \mid \cdot))$. Then, $H_{p}$ is a Hilbert space whose inner product norm coincides with the original norm on $A p$ ([5, p. 79]). By $\pi_{p}(a) x=a x$ for $a \in A, x \in H_{p}$ we can define an irreducible representation of $A$ on $H_{p}$, which is faithful since $A$ is prime. Therefore, $A$ is primitive. In addition, $\pi_{p}(\operatorname{soc}(A))=\mathscr{F}\left(H_{p}\right)$, the finite rank operators on $H_{p}$, and $\pi(K(A))=\mathscr{K}\left(H_{p}\right)([5, \mathrm{~F} .4 .3])$.

LEMMA 2.5. For every $\mathrm{C}^{*}$-algebra $A$ we have $\operatorname{soc}(A)=\operatorname{soc}(M(A))$ and $K(A)=$ $K(M(A))$.

Proof. If $p A p=\mathbb{C} p$, then $p A^{* *} p=\mathbb{C} p$, and thus $p M(A) p=\mathbb{C} p$. Therefore each minimal projection in $A$ is a minimal projection in $M(A)$, whence $\operatorname{soc}(A) \subseteq \operatorname{soc}(M(A))$. If $p \in M(A)$ is a minimal projection, then $\mathbb{C} p=p A p \subseteq A$, which implies that $p \in A$. Since, moreover, $M(A) p=A p$, we conclude that $\operatorname{soc}(M(A)) \subseteq \operatorname{soc}(A)$. The second assertion now follows by Proposition 2.1 (d).

REMARK. For Banach algebras there are also different notions of compact elements, most of which coincide in the $\mathrm{C}^{*}$-case (cf. [5, R.5]). For $\mathrm{W}^{*}$-algebras there is another concept of compact elements due to Breuer [6]. An element $a$ of a $\mathrm{W}^{*}$-algebra $A$ is called compact relative to $A$ if it belongs to the closed ideal $m(A)$ generated by all finite projections in $A$. In general, $m(A)$ is strictly larger than $K(A)$, and therefore less suited for our purposes.

3. Compact and weakly compact elementary operators. We begin our investigations by characterising the weakly compact elementary operators on the algebra $\mathscr{L}(H)$ as 
those which vanish on the Calkin algebra. Recall that a bounded linear operator $T$ on a Banach space $E$ is weakly compact if and only if the second adjoint $T^{* *}$ maps the second dual $E^{* *}$ of $E$ into the natural embedding $i E$ of $E$ into $E^{* *}([\mathbf{8}$, VI.4.2]). Recall also that the second dual $\mathscr{K}(H)^{* *}$ of the $\mathrm{C}^{*}$-algebra $\mathscr{K}(H)$ can be identified with $\mathscr{L}(H)$, and that an operator $T \in \mathscr{L}(\mathscr{L}(H))$ which leaves $\mathscr{K}(H)$ invariant is ultraweakly continuous if and only if $\left(T_{\mid \mathscr{C}(H)}\right)^{* *}=T$. For such $T$ denote by $\tilde{T} \in \mathscr{L}(\mathscr{C}(H))$ the induced operator on the Calkin algebra. The next lemma is a reformulation of [2, Proposition 2.1].

Lemma 3.1. Let $T: \mathscr{L}(H) \rightarrow \mathscr{L}(H)$ be an ultraweakly continuous bounded linear operator which leaves $\mathscr{K}(H)$ invariant. Then $T$ is weakly compact if and only if $\tilde{T}=0$.

Proof. If $T_{1}=T_{\mid \mathscr{F}(H)}$ denotes the restriction of $T$ to $\mathscr{K}(H)$ then, by Gantmacher's theorem ([8, VI.4.8]), $T_{1}$ is weakly compact if and only if $T_{1}^{* *}=T$ is weakly compact. By the remarks above, this is equivalent to $T_{1}^{* *} \mathscr{L}(H) \subseteq \mathscr{K}(H)$; i.e. $\tilde{T}=0$.

Now, we are in a position to apply Theorem 1.1 in order to unify Propositions 2.2, 2.3 and 2.7 of [2] (see also [19, Theorem 3.1] and [20, Theorem 3.1]) and to generalise them to arbitrary elementary operators.

Proposition 3.2. Let $A=\mathscr{L}(H)$, and for $a, b \in A^{n}$ let $S=\sum_{j=1}^{n} M_{a_{j} b_{j}}$ be the corresponding elementary operator. If the set $\left\{b_{1}, \ldots, b_{n}\right\}$ is linearly independent mod $\mathscr{K}(H)$ then the following conditions are equivalent:

(a) $S$ is weakly compact;

(b) $a_{j} \in \mathscr{K}(H)$ for all $1 \leqq j \leqq n$.

Proof. By Lemma 3.1, $S$ is weakly compact if and only if the induced operator $\tilde{S}$ vanishes on the Calkin algebra. Denoting by $\pi$ the canonical homomorphism from $\mathscr{L}(H)$ onto $\mathscr{C}(H)$ we have $\tilde{S}=\sum_{j=1}^{n} M_{\pi\left(a_{j}\right), \pi\left(b_{j}\right)}$. Since $\mathscr{C}(H)$ is prime, by Proposition 2.4 , it follows from Theorem 1.1 that $\tilde{S}=0$ if and only if $\pi\left(a_{j}\right)=0$ for all $j$.

In particular, 3.1 and 3.2 show that the primeness of $\mathscr{C}(H)$ is nothing but the assertion that $M_{a, b}$ is weakly compact if and only if $a$ or $b$ is compact. We recall at this place that $M_{a, b} \neq 0$ is compact on $\mathscr{L}(H)$ if and only if both $a$ and $b$ are compact ([16, Theorem 3]).

The following characterisation of weakly compact derivations on $\mathscr{L}(H)$ was first established by Akemann and Wright in [1, Theorem 3.1]. We obtain it here as an immediate consequence.

COROLLARY 3.3. The following conditions on a derivation $\delta$ on $\mathscr{L}(H)$ are equivalent:

(a) $\delta$ is weakly compact;

(b) $\delta \mathscr{L}(H) \subseteq \mathscr{K}(H)$;

(c) $\delta=\delta_{a}$ for some $a \in \mathscr{K}(H)$.

Proof. (a) $\Leftrightarrow$ (b) by 3.1 and (a) $\Leftrightarrow$ (c) by 3.2 . 
Our next aim is to extend Proposition 3.2 to arbitrary prime $C^{*}$-algebras. If $\pi$ is a representation of a $C^{*}$-algebra $A$, we denote by $\tilde{\pi}$ the extended ultraweakly continuous representation of its enveloping $\mathrm{W}^{*}$-algebra $A^{* *}$. Then $\bar{\pi}$ maps the closed unit ball $A_{1}^{* *}$ of $A^{* *}$ onto the closed unit ball $\pi(A)_{1}^{\prime \prime}$ of the ultraweak closure $\pi(A)^{\prime \prime}$. If we consider $A$ and $M(A)$ canonically embedded in $A^{* *}$ and $S=\sum_{j=1}^{n} M_{a_{j}, b_{j}}$ with $a_{j}, b_{j} \in M(A)$, then $S_{\bar{\pi}}=$ $\sum_{j=1}^{n} M_{\tilde{\pi}\left(a_{j}\right), \tilde{\pi}\left(b_{j}\right)}$ is the induced elementary operator on $\pi(A)^{\prime \prime}$.

Lemma 3.4. Let $A$ be a $C^{*}$-algebra and let $\pi$ be a representation of $A$. If $S$ is $a$ (weakly) compact elementary operator, then so is $S_{\bar{\pi}}$ and $S_{\bar{\pi}} \pi(A)^{\prime \prime} \subseteq \pi(A)$.

Proof. If $S$ is (weakly) compact, then so is its second adjoint $S^{* *}$ ([8], VI.4.8 and VI.5.2, respectively). From

$$
S_{\bar{\pi}} \pi(A)_{1}^{\prime \prime}=S_{\tilde{\pi}} \tilde{\pi}\left(A_{1}^{* *}\right)=\tilde{\pi} S^{* *} A_{1}^{* *} \subseteq \tilde{\pi}(A)=\pi(A)
$$

we obtain $S_{\bar{\pi}} \pi(A)^{\prime \prime} \subseteq \pi(A)$ as well as the (weak) compactness of $S_{\bar{\pi}}$.

LEMMA 3.5. If $S$ is a weakly compact elementary operator on a $\mathrm{C}^{*}$-algebra $A$, then $S A \subseteq K(A)$.

Proof. Let $\pi_{\mathrm{a}}$ denote the reduced atomic representation of $A$. Then $\pi_{\mathrm{a}}(A)^{\prime \prime}=$ $\sum_{t}^{\oplus} \mathscr{L}\left(H_{t}\right)([15,4.3 .8])$. If $S$ is weakly compact on $A$, by Lemma 3.4 its extension $S_{\tilde{\pi}}$ is weakly compact on $\pi_{\mathrm{a}}(A)^{\prime \prime}$. Denote by $S_{t}$ the induced mapping on $\pi_{t}(A)^{\prime \prime}=\mathscr{L}\left(H_{t}\right)$ for each $t \in \hat{A}$. Since $S_{t}$ is weakly compact it follows by Lemma 3.1 that $S_{t} \mathscr{L}\left(H_{t}\right) \subseteq \mathscr{K}\left(H_{t}\right)$. By an argument similar to [1, Lemma 3.2] (see also [2, Lemma 2.4]) there exists a sequence $\left(t_{n}\right)_{n \in \mathbb{N}}$ in $\hat{A}$ such that $S_{t}=0$ if $t \notin\left\{t_{n} \mid n \in \mathbb{N}\right\}$ and $\lim _{n \rightarrow \infty}\left\|S_{t_{n}}\right\|=0$. Therefore, if $y=S_{\bar{\pi}_{\mathrm{a}}} x$ for some $x=\left(x_{t}\right) \in \pi_{\mathrm{a}}(A)^{\prime \prime}$, then $y_{t}=0$ for all $t \notin\left\{t_{n} \mid n \in \mathbb{N}\right\}$ and $\lim _{n \rightarrow \infty}\left\|y_{t_{n}}\right\|=0$. From this and Lemma 3.4 we obtain

$$
S_{\tilde{\pi}_{\mathrm{a}}} \sum_{t}^{\oplus} \mathscr{L}\left(H_{t}\right) \subseteq \sum_{t}^{\oplus} \mathscr{K}\left(H_{t}\right) \cap \pi_{\mathrm{a}}(A)
$$

Our last claim is that

$$
\sum_{t}^{\oplus} \mathscr{K}\left(H_{t}\right) \cap \pi_{\mathrm{a}}(A)=\pi_{\mathrm{a}}(K(A))
$$

This together with (1) will yield $S_{\bar{\pi}_{\mathrm{a}}} \pi_{\mathrm{a}}(A)^{\prime \prime} \subseteq \pi_{\mathrm{a}}(K(A))$ from which $S A \subseteq K(A)$ follows by the faithfulness of $\pi_{\mathrm{a}}([\mathbf{1 5}, 4.3 .11])$.

Since $M_{a, a}$ is compact for every $a \in K(A)$, by (1),

$$
M_{\pi_{\mathrm{a}}(a), \pi_{\mathrm{a}}(a)} \pi_{\mathrm{a}}(A)^{\prime \prime} \subseteq \sum_{t}^{\oplus} \mathscr{K}\left(H_{t}\right)
$$

In particular, $\pi_{a}(a) \pi_{a}(a)^{*} \pi_{a}(a)$ belongs to $\sum_{t}^{\oplus} \mathscr{K}\left(H_{t}\right)$; i.e. $\lim _{n \rightarrow \infty}\left\|\pi_{t_{n}}(a)\right\|^{3}=0$ for some 
sequence $(t(n))_{n \in \mathbb{N}}$ in $\hat{A}$ and $\left\|\pi_{t}(a)\right\|^{3}=0$ for all $t \notin\{t(n) \mid n \in \mathbb{N}\}$. Thus $\pi_{\mathrm{a}}(a) \in \sum_{t}^{\oplus} \mathscr{K}\left(H_{t}\right)$. Conversely, take $a \in A$ with $\pi_{\mathrm{a}}(a) \in \sum_{t}^{\oplus} \mathscr{K}\left(H_{t}\right)$, i.e. $\pi_{t}(a)=0$ for all but a countable number of indices $t(n) \in \hat{A}, \lim _{n \rightarrow \infty}\left\|\pi_{t(n)}(a)\right\|=0$ and $\pi_{t(n)}(a) \in \mathscr{K}\left(H_{t(n)}\right)$ for all $n \in \mathbb{N}$. Since the multiplications $M_{\pi_{t_{(n)}(a), \pi_{t(n)}(a)}}$ are compact on $\mathscr{L}\left(H_{t(n)}\right)$, the formula

$$
S_{m}=\bigoplus_{n=1} M_{\pi_{t(n)}(a), \pi_{(n)}(a)}
$$

defines a sequence of compact mappings which converges uniformly to $M_{\pi_{\mathrm{a}}(a), \pi_{\mathrm{a}}(a)}$, as we

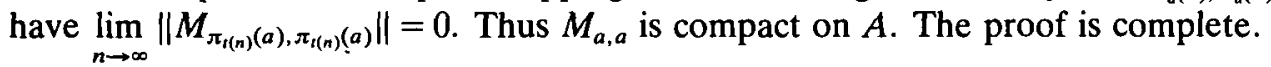

Remark. The conclusion in Lemma 3.5 cannot be reversed as the example $A=\mathscr{K}(H)$ and $S=M_{p, p}$, with $p$ an infinite dimensional projection on $H$, shows.

In [9], Fong and Sourour conjectured that on the Calkin algebra $\mathscr{C}(H)$ on a separable Hilbert space $H$ there cannot exist any non-zero compact elementary operator. This conjecture was confirmed by Apostol and Fialkow [3], and independently by Magajna [11]. Propositions 2.3 and 2.4 show that this is only a special case of the following consequence of Lemma 3.5.

COROLlARY 3.6. There are no non-zero weakly compact elementary operators on a $\mathrm{C}^{*}$-algebra without non-zero compact elements.

Before we go on to establish our main results, we provide the proof of Proposition 2.1 that was promised in Section 2.

Proof of 2.1. (a) $\Leftrightarrow$ (b) by definition, and (b) $\Rightarrow$ (c) is obvious.

(c) $\Rightarrow$ (a) Since $M_{a, a}$ weakly compact implies that $a A a \subseteq K(A)$, by Lemma 3.5 , it follows that $a \in K(A)$.

(b) $\Leftrightarrow$ (d) by [5, $\left.C^{*} .1 .3\right]$.

(a) $\Leftrightarrow$ (f) by identity (2) in the proof of Lemma 3.5 .

(f) $\Rightarrow$ (e) If $a \in A$ is such that $\pi_{\mathrm{a}}(a) \in \sum_{t}^{\oplus} \mathscr{K}\left(H_{t}\right)$, then there is a sequence $\left(t_{n}\right)_{n \in \mathbb{N}}$ in $\hat{A}$ with $\lim _{n \rightarrow \infty}\left\|\pi_{t_{n}}(a)\right\|=0$ and $\pi_{t}(a)=0$ if $t \notin\left\{t_{n} \mid n \in \mathbb{N}\right\}$. If $a \neq 0$ we may assume that $\pi_{t_{n}}(a) \neq 0$, and thus $\mathscr{K}\left(H_{t_{n}}\right) \cap \pi_{t_{n}}(A) \neq 0$. By Kadison's transitivity theorem, it follows that $\mathscr{K}\left(H_{t_{n}}\right) \subseteq \pi_{t_{n}}(A)$ (cf. $[15,6.1 .4]$ ). Hence $a \in \sum_{n}^{\oplus} I_{n}$, where $I_{n} \cong \mathscr{K}\left(H_{t_{n}}\right)$ via $\pi_{\mathrm{a}}$ for each $n \in \mathbb{N}$. An argument analogous to the last part in the proof of Lemma 3.5 yields (e) $\Rightarrow$ (b).

Using the results obtained so far, it is now easy to characterise the weakly compact elementary operators on a prime $\mathrm{C}^{*}$-algebra. 
THEOREM 3.7. Let $A$ be a prime $C^{*}$-algebra and for $a, b \in M(A)^{n}$ let $S=\sum_{j=1}^{n} M_{a_{j}, b_{j}}$ be the corresponding elementary operator. If the set $\left\{b_{1}, \ldots, b_{n}\right\}$ is linearly independent $\bmod K(A)$ then the following conditions are equivalent:

(a) $S$ is weakly compact;

(b) $a_{j} \in K(A)$ for all $1 \leqq j \leqq n$.

Proof. (b) $\Rightarrow$ (a) This is true in every $\mathrm{C}^{*}$-algebra and without the additional assumption on $\left\{b_{1}, \ldots, b_{n}\right\}$. If $a_{j} \in K(A)$ for all $j$, then the left multiplication $L_{a_{i}}$ is weakly compact for all $j([20$, Theorem 3.1$])$. Since the weakly compact operators form an ideal ([8, VI.4.6]), the operator $S=\sum_{j=1}^{n} L_{a_{j}} R_{b_{j}}$ is weakly compact.

(a) $\Rightarrow$ (b) First assume that $K(A)=0$. By Lemma $3.5, S$ is weakly compact if and only if $S=0$. Thus the assertion follows by Theorem 1.1. Now assume $K(A) \neq 0$. If $p$ is a minimal projection in $A$, then the corresponding irreducible representation $\left(\pi_{p}, H_{p}\right)$ of $A$ (see Section 2) extends to an irreducible representation of $M(A)$, denoted by the same symbol. Since $M(A)$ is prime ([13, Lemma 2.2]), $\pi_{p}$ is faithful on $M(A)$. By hypothesis, the set $\left\{\pi_{p}\left(b_{1}\right), \ldots, \pi_{p}\left(b_{n}\right)\right\}$ is linearly independent $\bmod \mathscr{K}\left(H_{p}\right)$ (which equals $\pi_{p}(K(A))$ by Lemma 2.5). Since $S_{\tilde{\pi}_{p}}=\sum_{j=1}^{n} M_{\pi_{p}\left(a_{j}\right), \pi_{p}\left(b_{j}\right)}$ is weakly compact on $\mathscr{L}\left(H_{p}\right)$ by Lemma 3.4, it follows that $\pi_{p}\left(a_{j}\right) \in \mathscr{K}\left(H_{p}\right)$ by Proposition 3.2. Thus $a_{j} \in K(A)$ for all $1 \leqq j \leqq n$.

The following description of compact elementary operators was proved in the case $A=\mathscr{L}(H)$ by Fong and Sourour in [9, Theorem 2].

THEOREM 3.8. Let $A$ be a prime $\mathrm{C}^{*}$-algebra and for $a, b \in M(A)^{n}$ let $S=\sum_{j=1}^{n} M_{a_{j}, b_{j}}$ be the corresponding elementary operator. If $S$ is compact and $\left\{b_{1}, \ldots, b_{n}\right\}$ is linearly independent, then $a_{j} \in K(A)$ for all $1 \leqq j \leqq n$.

Proof. If $K(A)=0$, then $S$ is compact if and only if $S=0$ by Lemma 3.5. Hence, the assertion is a consequence of Theorem 1.1.

If $K(A) \neq 0$, then $K(A)=\pi^{-1}(\mathscr{K}(H))$ for some faithful irreducible representation $(\pi, H)$ of $A$. Since $S$ is compact if and only if the induced elementary operator $S_{\bar{\pi}}$ on $\mathscr{L}(H)$ is compact (Lemma 3.4), the assertion follows from the corresponding result on $\mathscr{L}(H)([9$, Theorem 2]).

Corollary 3.9. A non-zero elementary operator $S$ on a prime $\mathrm{C}^{*}$-algebra $A$ is compact if and only if there are linearly independent subsets $\left\{a_{1}, \ldots, a_{n}\right\},\left\{b_{1}, \ldots, b_{n}\right\}$ in $K(A)$ such that $S=\sum_{j=1}^{n} M_{a_{j}, b_{j}}$.

Proof. Let $n=l(S) \geqq 1$ be the minimal length of $S$. If $S=\sum_{i=1}^{n} M_{a_{j}, b_{i}}$ is a minimal representation, both subsets $\left\{a_{1}, \ldots, a_{n}\right\}$ and $\left\{b_{1}, \ldots, b_{n}\right\}$ of $M(A)$ are linearly 
independent (see Section 1). If $S$ is compact, so is $\bar{S}=\sum_{j=1}^{n} M_{b_{j}^{*}, a_{j}^{*}}$ since $\bar{S} x=\left(S x^{*}\right)^{*}$. Thus, by Theorem $3.8,\left\{a_{1}, \ldots, a_{n}\right\} \subseteq K(A)$ and $\left\{b_{1}, \ldots, b_{n}\right\} \subseteq K(A)$.

The converse conclusion follows from Vala's theorem ([16, Theorem 3]) stating that $M_{a, b}$ is compact whenever $a$ and $b$ are compact operators on $H$ applied in an irreducible representation $(\pi, H)$ of $A$.

As another consequence of Theorem 3.8, there are no non-zero compact derivations on an infinite dimensional prime $\mathrm{C}^{*}$-algebra. This result appeared (with an incorrect proof) for the case $A=\mathscr{L}(H)$ in [10]. Similarly, Corollary 3.3 can be extended to prime $\mathrm{C}^{*}$-algebras.

COROLLARY 3.10. Every compact elementary operator on a prime $\mathrm{C}^{*}$-algebra is the norm-limit of elementary operators of finite rank.

Proof. Let $S$ be a compact elementary operator on the prime $\mathrm{C}^{*}$-algebra $A$. Without loss of generality we may assume that $l(S)=1$. By Corollary 3.9 , we thus have $S=M_{a, b}$ with $a, b$ in $K(A) \backslash\{0\}$. Since

$$
M_{a, b}=\frac{1}{4} \sum_{k=0}^{3} i^{k} M_{\left(b+i^{k} a^{*}\right)^{*},\left(b+i^{k} a^{*}\right)},
$$

we may further assume that $a=b^{*}$. Since $K(A)=\overline{\operatorname{soc}(A)}$, there is a sequence $\left(b_{n}\right)_{n \in \mathbb{N}}$ in $\operatorname{soc}(A)$ with $b=\lim _{n \rightarrow \infty} b_{n}$. Similarly to [5, $\left.\mathrm{C}^{*} .1 .2\right]$ one can show that each multiplication $M_{b_{n}^{*}, b_{n}}$ is of finite rank. Therefore, $M_{b^{*}, b}=\lim _{n \rightarrow \infty} M_{b_{n}^{*}, b_{n}}$ is approximated by finite rank operators.

\section{REFERENCES}

1. C. A. Akemann and S. Wright, Compact and weakly compact derivations of $\mathrm{C}^{*}$-algebras, Pacific J. Math. 85 (1979), 253-259.

2. C. A. Akemann and S. Wright, Compact actions on $\mathrm{C}^{*}$-algebras, Glasgow Math. J. 21 (1980), 143-149.

3. C. Apostol and L. A. Fialkow, Structural properties of elementary operators, Canad. J. Math. 38 (1986), 1485-1524. 25-38.

4. R. J. Archbold, On factorial states of operator algebras, J. Functional Analysis 55 (1984),

5. B. A. Barnes, G. J. Murphy, M. R. F. Smyth and T. T. West, Riesz and Fredholm theory in Banach algebras, Pitman Research Notes in Mathematics 67 (1982). $243-254$.

6. M. Breuer, Fredholm theories in von Neumann algebras I, Math. Ann. 178 (1968),

7. J. Dixmier, Les C*-algèbres et leurs représentations, (Gauthier-Villars, 1969).

8. N. Dunford and J. T. Schwartz, Linear operators Part I, (Interscience, New York, 1958).

9. C. K. Fong and A. R. Sourour, On the operator identity $\sum A_{k} X B_{k} \equiv 0$, Canad. J. Math. 31 (1979), 845-857.

10. Y. Ho, A note on derivations, Bull. Inst. Math. Acad. Sinica 5 (1977), 1-5.

11. B. Magajna, A system of operator equations, Canad. Math. Bull. 30 (1987), 200-209. 
12. W. S. Martindale, Prime rings satisfying a generalized polynomial identity, J. Algebra 12 (1969), 576-584.

13. M. Mathieu, Elementary operators on prime $\mathrm{C}^{*}$-algebras, $\mathrm{I}$, to appear.

14. M. Mathieu, Applications of ultraprime Banach algebras in the theory of elementary operators, Thesis, (Tübingen, 1986).

15. G. K. Pedersen, $C^{*}$-algebras and their automorphism groups, (Academic Press, 1979). (1964).

16. K. Vala, On compact sets of compact operators, Ann. Acad. Sci. Fenn. Ser. A I 351

17. K. Vala, Sur les éléments compacts d'une algèbre normée, Ann. Acad. Sci. Fenn. Ser. A I 407 (1967).

18. K. Ylinen, Compact and finite-dimensional elements of normed algebras, Ann. Acad. Sci. Fenn. Ser. A I 428 (1968).

19. K. Ylinen, Dual $C^{*}$-algebras, weakly semi-completely continuous elements, and the extreme rays of the positive cone, Ann. Acad. Sci. Fenn. Ser. A I 599 (1975).

20. K. Ylinen, Weakly completely continuous elements of $\mathrm{C}^{*}$-algebras, Proc. Amer. Math. Soc. 52 (1975), 323-326.

MATHEMATISChES INSTITUT DER

UNIVERSITAT TÜBINGEN

7400 TÜBINGEN

West Germany 